\title{
Why is EIT so hard, and what are we doing about it?
}

\author{
Andy Adler ${ }^{1}$, Bartłomiej Grychtol ${ }^{2}$ and Richard Bayford ${ }^{3}$ \\ ${ }^{1}$ Systems and Computer Engineering, Carleton University, Ottawa, K1S 5B6, \\ Canada \\ ${ }^{2}$ Fraunhofer Project Group for Automation in Medicine and Biotechnology, \\ Mannheim, Germany \\ ${ }^{3}$ Department of Natural Sciences, Middlesex University, London, UK \\ E-mail: adler@sce.carleton.ca
}

This focus issue of Physiological Measurement follows the successful $15^{\text {th }}$ International Conference on Biomedical Applications of Electrical Impedance Tomography (EIT2014) held at the Glen House Resport in Gananoque, Ontario, Canada from April 24-26, 2014. The conference was organized by Andy Adler, of the department of systems and computer engineering at Carleton University, in Ottawa, Canada, and co-organized by Bartłomiej Grychtol, of the Fraunhofer Project Group for Automation in Medicine and Biotechnology in Mannheim, Germany. A new award for best student paper was presented to Robert Winkler (Winkler et al 2014), and runner-up award to Melody Dodd (Dodd and Mueller 2014).

This continues the tradition of successful conferences on biomedical applications of electrical impedance tomography, as was the case with the $14^{\text {th }}$ International Conference on Biomedical Applications of Electrical Impedance Tomography (EIT2013), held on 22-25 April 2013 at Heilbad Heiligenstadt, Germany, and hosted by Uwe Pliquet of the Institut für Bioprozess- und Analysenmeßtechnik. This year's conference is the XVIth International Conference on Biomedical Applications of Electrical Impedance Tomography (EIT2015) to be held in Neuchâtel, Switzerland on 2-5 June 2015, and hosted by Josep Solà and Fabian Braun of the Centre suisse d'électronique et de microtechnique. This conference will be followed by a focus issue in Physiological Measurement that will be published in 2016 .

This issue contains papers stemming from discussion and feedback during the 2014 conference, and is also an opportunity for new researchers to join the community and describe recent innovations. There were 77 accepted submissions (including three keynotes, 45 oral presentation, and 29 posters). All authors were invited to prepare new peer-reviewed papers for inclusion in this issue of Physiological Measurement. Manuscripts were put through a process of careful review before selection, and 18 were accepted (of 27 submitted), covering an important range of topics. 


\section{Electrical Impedance Tomography}

EIT images the impedance within a body from electrical stimulations and measurements on its surface. It shows promise for thoracic, brain, breast, abdominal and prostate imaging.

Those of us working in EIT have written an introductory paragraph like the previous one, many, many times. Each time we write it, we try to compress the text, just a little more. Why? We are looking forward to the day when there is no longer a need to explain EIT; when there is no longer the need to call it a promising, "new" technology. Instead, we would like to write, "Based on evidence of improved patient outcomes and safety, EIT devices are increasingly used ...", and provide the references to prove it. This editorial reviews how far along this road we have come.

\section{Why is EIT so hard?}

The question "Why is EIT so hard?" (Lionheart et al 2005) is an important one, because EIT appears to be deceptively simple. The design of relatively low frequency $(\leq 250 \mathrm{kHz})$ electronics is well understood and the components are cheap. Body surface electrodes have been used routinely for over a hundred years. And many physiological processes of interest introduce conductivity contrasts into the body. The lungs move low conductivity air; the heart (and circulation defects like a haemorage) moves high conductivity blood; cancerous tissue has a different impedance spectrum due to changes in cell structures and angiogenesis; neural activity changes cell membrane permeability, and thus the conductivity; gastric motility moves a conductivity contrasting meal bolus.

However, this apparent ease is deceptive: a research group's first designs of EIT electronics are rarely optimal, and a new application of EIT typically shows it to be more sensitive to many effects other than the physiology of interest. EIT is an ill-conditioned inverse problem because electric currents propagate in a diffusive way within a body and thus tend to stay near the stimulation electrodes (as opposed to, for example, X-rays). This implies two things about EIT:

(i) Low sensitivity to the phenomena of interest. We are typically interested in conductivity changes at depth, but relatively little of the current flows deeply into the body. This effect is made worse by several effects. In chest EIT, current propagates in 3D from small, ECG-type, electrodes. Early EIT algorithms were calculated in 2D, but, in 3D, the sensitivity in the centre of the chest decreases by 1.6 (it would be possible to address this with large vertical electrode strips, but this has been rarely done). Chest EIT has, for historical reasons, used adjacent simulation and measurement, which has very poor depth sensitivity. For 16 electrodes, the adjacent simulation and measurement is 24 times less sensitive in the chest centre than next-to-opposite patterns (Adler et al 2011, Bayford et al 1996). Additionally, the low-conductivity lungs act like a shield to discourage current from penetrating to the centre. Normal lung conductivity decreases the sensitivity in the medium 
center by 2.8 from the case where the chest has uniform conductivity. $\neq$ This effect is increased by the fat underneath the skin of many patients (but this, most commonly, is not an issue when EIT is tested on healthy young graduate students). Other applications of EIT have similar challenges: for example, brain EIT must make measurements through the low conducivity of the skull.

(ii) High sensitivity to effects which are not of interest. EIT is very sensitive to any imperfections in the electronics hardware and the electrode contact. Since the impedance differences of interest typically produce changes in measurements on the order of $0.1 \%(60 \mathrm{~dB})$ then the required instrumentation must be better than this. This is a challenging requirement for the amplifiers alone. While instrumentation amplifiers with specifications (for common-mode rejection ratio, for instance) of $100 \mathrm{~dB}$ are available, their performance degrades significantly above about $1 \mathrm{kHz}$. Furthermore, the entire instrumentation chain must achieve this performance. Temperature changes, crosstalk between wires, electromagnetic interference, and the effects of finite output impedance of the current sources with the variabilities in electrode contact impedance introduce variability in signals.

The difficulties produced by the electrodes should be underlined. The electrode positions change with posture and breathing, and the electrode contact changes with patient sweat and, over time, as the contact gel dries. A further difficulty in modelling the electrodes has resulted from an unfortunate misunderstanding between the hardware and mathematical EIT communities. The term "contact impedance" $\left(z_{c}\right)$ has been used to describe both: 1) surface impedance of the electrode material (which allows current to pass through the electrode rather than the body surface in which it is in contact), and 2) the equivalent input impedance which the current source must drive into the body. These are clearly not the same; reconstruction algorithms should account for both effects, but we are not aware of any that do.

Thus the mathematical challenge of EIT reconstruction. Algorithms need to calculate images of the effects of interest, and reject the artefacts from any other, confounding, effects. A good algorithm should have a spatially uniform response. Conductivity contrasts outside of the field of measurement (i.e. gas in the intestines for a chest EIT system), should not influence the reconstructed images. Furthermore, reconstruction algorithms should be robust, and provide some indication of the reliability and error bounds on the calculated images. These desirata are clearly challenging.

Most biomedical applications of EIT use difference imaging to calculate an impedance change image from a change in measurements, either over time (giving time difference EIT, tdEIT) or stimulation frequency (giving frequency difference EIT, fdEIT). Difference EIT is less sensitive to experimental uncertainties that do not change (much) between measurements, such as posture, electrode contact, or temperature. However, clearly, as EIT examinations are prolonged (hours or days) the assumptions

$\ddagger$ Software to calculate these values available at: eidors.org/tutorial/netgen/signals.shtml 
of tdEIT are less valid. Interestingly, geophysical applications of electrical resistance tomography (the equivalent technique) more commonly use absolute imaging. This may be because objects of interest are relatively closer to the surface (compared to the electrode spacing), processes of interest are much slower, or because very low stimulation frequencies are used, at which electronic imperfections are less serious.

One last challenge for EIT is that we rarely care about tissue impedance (or conductivity) by itself, although it affects difference images (Grychtol and Adler 2013). The clinical need is to measure air flow or identify cancerous tissue. In many cases, the relationship between the physiology of interest and the tissue impedance is nonlinear and poorly understood. Fortunately, experimental studies have often shown that conductivity change is proportional to (for example) air volume change over a relatively wide range (e.g. Adler et al 1997).

\section{What are we doing about it?}

The list of challenges in the previous section may seem long and its effect discouraging. However, the EIT research community is vibrant and is developing many novel approaches to manage the difficulties. Using novel experimental protocols and analysis methodologies, EIT researchers have been working to provide robust, unique and valuable clinical information. How are we doing this?

First, by carefully understanding EIT's sensitivity and limitations. In order to correctly interpret EIT images, it is important to understand the factors which influence the images. And, next, by novel measurement protocols and analysis methodologies. Such novel approaches focus the effort on those clinical and experimental applications where EIT would be the most reliable.

In this focus issue, we note numerous studies which seek to understand the physiological origins of EIT signals. Proença et al (2015) seek to determine the influence of heart motion in the EIT-based estimation of cardiac output, Gagnon et al (2015) investigate the aliasing produced by rapid EIT signals, Krueger-Ziolek et al (2015) measure the influence of the position of the chest EIT electrode plane, Biguri et al (2015) model the effect of boundary movements for various reconstruction algorithms and Becher et al (2015) investigate the influence of tidal volume on ventilation inhomogeneities.

Several studies seek to improve algorithms and optimize measurements configurations. Braun et al (2015) seek to optimize EIT-based measurement of the aortic signal, Antink et al (2015) develop a shape-based quality evaluation and reconstruction method, Jang et al (2015) develop techniques to detect admittivity anomalies in fdEIT, Zhou et al (2015) compare algorithms using a total-variation functional, Roth et al (2015) investigate the correlation between ventilation and lung electrical properties, and Gaggero et al (2015), report on a robust test framework which may be used to calibrate and compare EIT systems.

Another group of papers investigates the sensitivity with which EIT can determine 
specific physiological phenomena. Aristovich et al (2015) investigate the signals and potential artefacts from evoked neural activity, Nebuya et al (2015) investigate EIT lung density values for diagnosis, and Dowrick et al (2015) conduct in-vivo measurements of healthy and ischaemic rat brains in order to understand imaging of strokes. Two papers investigate measures of perfusion, including a regional ventilation-perfusion index (Muller et al 2015) and the redistribution of perfusion after embolism (Nguyen et al 2015). Novel experimental configurations are also introduced, including a rotary planar electrode array (Zhang et al 2015), and a frequency selective common feedback design (Langlois et al 2015).

Considering the studies in this focus issue, we can see a clear maturation of EIT research. There is now proportionately less focus on the mechanics of image reconstruction, but rather on the analysis of EIT data and images. In order to suggest a unifying framework in which to understand these various analysis steps, we present an analysis "pipeline" (figure 1) (thanks to Inéz Frerichs for help elaborating this concept). From left to right, EIT data are processed from the application of electrodes to the generation of application specific measures, such as the centre of ventilation $(\mathrm{CoV})$. Image reconstruction plays a key role in the analysis, but many other steps are critical to producing robust measures that are clinically relevant. Currently, we are seeing many innovations in functional EIT (fEIT) analysis, where tissue types are distinguished based on their different response characteristics in the EIT waveforms.
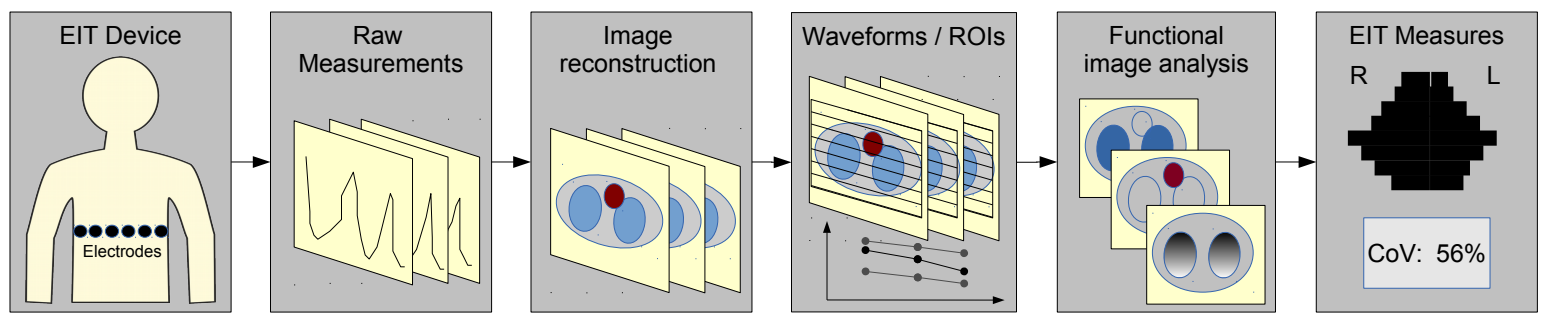

Figure 1. Analysis "pipeline" for EIT data. From left to right, an EIT device is placed on the subject, raw data acquired, each frame of data is reconstructed, and regions of interest (ROIs) and waveforms in the data identified. From these waveforms functional EIT images are generated, and application specific EIT measures calculated.

\section{Perspectives}

When discussing EIT's potential, one joke has been made, referring to the frustrations of a technology that shows much promise and so many challenges: "it doesn't clearly work, so we can reap the fruits of its images, or not work, so we can change direction; it usually almost works, which is an incitement to redouble our efforts." (Holder, 2005). Given a short summary of "Why is EIT so hard?" and a look at "What we (the EIT community) are doing about it?", we see an exciting movement to conduct the research to address the challenges. 
Recent review papers have noted a steady increase in scientific and medical interest in EIT (e.g. Frerichs et al 2014). In Adler et al (2012), this trend was explained by the increasing clinical "draw". In the early days of EIT, the lung was widely assumed to behave as a relatively uniform system. There was thus less interest in an imaging technology to characterize such a simple system. However, throughout the 1990s, there was an increased understanding of the complexity of the lungs, the difficulties in providing safe and adequate ventilatory support, and the clinical consequences (ventilator induced/associated lung injury - VILI/VALI). The increasing awareness of this problem drove an interest in strategies to address it (Lung protective ventilation LPV), and a technology to monitor it (EIT). In a graph of the number of publications per year of on these topics, one can see a sudden increase in interest in the clinical problem (VILI/VALI) in about 1997, followed by an interest in strategies to address it (LPV and EIT) approximately five years later (figure 2).

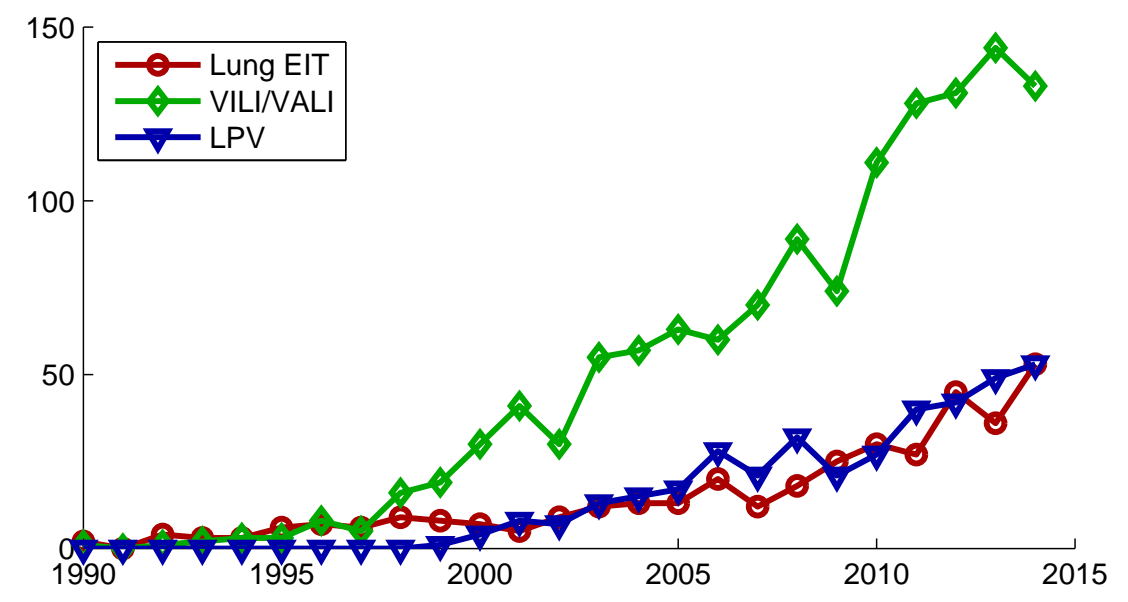

Figure 2. Number of publications vs. publication date on VILI/VALI (Ventilator Induced/Associated Lung Injury), LPV (Lung Protective Ventilation) and lung - chest - thoracic EIT published in peer-reviewed journals in Medline. (Source: ISI Web of Knowledge, Thomson Reuters, New York, USA.)

Looking at the papers in this focus issue, and at the state of the EIT community, we observe strong reasons for optimism.

- Scientific interest in EIT is strong and growing. Within its traditional areas of interest there is increasing appreciation of the unique information it can provide. We see improving collaboration between mathematicians, engineers, physiologists and clinicians; and also interaction with the corresponding geophysical community. Novel image reconstruction and analysis approaches are proposed, incorporating deep mathematical insights and concern for the challenges of EIT data. Data and algorithms are being shared more often between experimental and theoretical research groups.

- Commercial devices are now available. Two companies (both sponsors of the EIT 2014 and EIT 2015 conferences) sell EIT devices which are approved for human 
use. This will help address several limitations which have kept many researchers from using EIT: the difficulty in obtaining EIT devices, and the difficulty in making clinical measurements on patients.

- Medical results are promising. Much of the earliest EIT work was naturally concerned with validation of the technology. It was important to show that EIT gave compatible results to that from gold standards, such as CT. EIT studies were also almost always retrospective; data were analysed afterwards to verify whether the information from EIT was accurate. EIT is now beginning to move beyond the stage of validation to other gold standards, and is being used for prospective interventions (e.g. Wolf et al 2013). We have evidence that EIT can predict other clinically important events, such as decreases in arterial oxygenation, and to detect physiological conditions for which no other technology is available.

The review paper concluded with two suggestions: (1) think about the physiology; for example, an EIT indication "my patient's lungs are 30\% collapsed" may provide deep insights which lead to therapeutic interventions; and (2) analyse EIT images in creative ways, [using] sophisticated signal processing algorithms to generate new parameters. When we look at the interesting collection of papers for this focus issue, we feel optimistic. Many novel insights and creative ways to acquire and analyse EIT are proposed. We will soon be able to write?

EIT is a promising lifesaving technology ...

\section{References}

Adler A, Amato MB, Arnold JH, Bayford R, Bodenstein M, Böhm SH, Brown BH, Frerichs I, Stenqvist O, Weiler N, Wolf GK, "Whither lung EIT: where are we, where do we want to go, and what do we need to get there?", Physiol Meas, 33:679-694, 2012.

Adler A, Gaggero PO, Maimaitijiang Y, "Adjacent Stimulation and Measurement Patterns Considered Harmful" Physiol Meas, 32:731-744, 2011.

Adler A, Amyot R, Guardo R, Bates JHT, Berthiaume Y "Monitoring changes in lung air and liquid volumes with electrical impedance tomography" J Applied Physiology 83:1762-1767, 1997

Antink CH, Pikkemaat R, Leonhardt S "A shape-based quality evaluation and reconstruction method for electrical impedance tomography", Physiol Meas, 2015:???

Aristovich KY, dos Santos GS, Holder DS "Investigation of potential artefactual changes in measurements of impedance changes during evoked activity: implications to electrical impedance tomography of brain function" Physiol Meas, 2015:???

Bayford RH, Boone KG, Hanquan Y and Holder DS, ".mprovement of the positional accuracy of EIT images of the head using a Lagrange multiplier reconstruction algorithm with diametric excitation" Physiol Meas, 17:A49-A57, 1996

Becher T, Kott M, Schädler D, Vogt B, Meinel T, Weiler N, Frerichs I, "Influence of tidal volume on ventilation inhomogeneity assessed by electrical impedance tomography during controlled mechanical ventilation", Physiol Meas, 2015:???

Biguri A, Adler A, Grychtol B, Soleimani M "Tracking boundary movement and exterior shape modelling in lung EIT imaging" Physiol Meas, 2015:???

Braun F, Proença M, Rapin M, Lemay M, Adler A, Grychtol B, Solá J, Thiran J-P "Aortic blood pressure measured via EIT: investigation of different measurement settings", Physiol Meas, 2015:??? 
Dodd M, Mueller JL, "Fast D-bar reconstructions of ventilation and perfusion on a pairwise current injection system" XV Int Conf biomedical Appl EIT, p. 81, Gananoque, Canada, Apr 24-26, 2014

Dowrick T, Blochet C, Holder DH, "In-vivo bioimpedance measurement of healthy and ischaemic rat brain; implications for stroke imaging using electrical impedance tomography", Physiol Meas, 2015:???

Frerichs I, Becher T, Weiler N. "Electrical impedance tomography imaging of the cardiopulmonary system" Curr Opin Crit Care 20:323-332, 2014

Gaggero PO, Adler A, Waldmann AD, Mamatjan Y, Justiz J, Koch VM, "Automated robust test framework for electrical impedance tomography", Physiol Meas, 2015:???

Gagnon H, Grychtol B, Adler A, "A comparison framework for temporal image reconstructions in electrical impedance tomography", Physiol Meas, 2015:???

Grychtol B, Adler A, "Uniform background assumption produces misleading lung EIT images" Physiol Meas, 34:579-593, 2013.

Holder DS (Ed.), Electrical Impedance Tomography Institute of Physics Publishing, Bristol and Philadelphia. 2005

Jang J, Seo JK "Detection of admittivity anomaly on high-contrast heterogeneous backgrounds using frequency difference EIT", Physiol Meas, 2015:???

Krueger-Ziolek S, Schullcke B, Kretschmer J, Müller-Lisse U, Möller K, Zhao Z, "Positioning of electrode plane systematically influences EIT imaging", Physiol Meas, 2015:???

Langlois P, Wu Y, Bayford RH, Demosthenous A "On the application of frequency selective common mode feedback for multifrequency EIT", Physiol Meas, 2015:???

Lionheart WRB, Polydorides N, Borsic A, "The Reconstruction Problem William", in Holder DS (Ed.), Electrical Impedance Tomography Institute of Physics Publishing, Bristol and Philadelphia. 2005

Muller PA, Li T, Isaacson D, Newell JC, Saulnier CJ, Kao T-J, Ashe J "Estimating a regional ventilation-perfusion index" Physiol Meas, 2015:???

Nebuya S, Koike T, Imai H, Iwashita Y, Brown BH, Soma K "Feasibility of using lung density values estimated from EIT images for clinical diagnosis of lung abnormalities in mechanically ventilated ICU patients", Physiol Meas, 2015:???

Nguyen DT, Bhaskaran A, Chik W, Barry MA, Pouliopoulos J, Kosobrodov R, Jin C, Oh TI, Thiagalingam A, McEwan AL "Perfusion redistribution after a pulmonary-embolism-like event with contrast enhanced EIT", Physiol Meas, 2015:???

Proença M, Braun F, Rapin M, Solà J, Adler A, Grychtol B, Bohm S, Lemay M, Thiran J-P, "Influence of heart motion on cardiac output estimation by means of electrical impedance tomography: a case study", Physiol Meas, 2015:???

Roth CJ, Ehrl A, Becher T, Frerichs I, Schittny JC, Weiler N, Wall WA "Correlation between alveolar ventilation and electrical properties of lung parenchyma", Physiol Meas, 2015:???

Winkler R, Staboulis S, Rieder S, Hyvönen N, "Fine-tuning of the Complete Electrode Model" XV Int Conf biomedical Appl EIT, p 28, Gananoque, Canada, Apr 24-26, 2014

Wolf GK, Gómez-Laberge C, Rettig JS, Vargas SO, Smallwood GD, Prabhu SP, Vitali SH, Zurakowski D, Arnold JH, "Mechanical Ventilation Guided by Electrical Impedance Tomography in Experimental Acute Lung Injury" Crit care med, 41:1296-1304, 2013.

Zhang X, Chatwin C, Barber DC, "A feasibility study of a rotary planar electrode array for electrical impedance mammography using a digital breast phantom", Physiol Meas, 2015:???

Zhou A, dos Santos GS, Dowrick T, Avery J, Sun Z, Xu H, Holder DS "Comparison of total variation algorithms for electrical impedance tomography", Physiol Meas, 2015:??? 